\title{
Semi-field evaluation of freestanding transfluthrin passive emanators and the BG sentinel trap as a "push-pull control strategy" against Aedes aegypti mosquitoes
}

Mgeni M. Tambwe ${ }^{1,2,3^{*}}$, Sarah J. Moore ${ }^{1,2,3}$, Hassan Chilumba'1, Johnson K. Swai', Jason D. Moore ${ }^{1,2}$, Caleb Stica' and Adam Saddler 1,2,3

\begin{abstract}
Background: Spatial repellents that drive mosquitoes away from treated areas, and odour-baited traps, that attract and kill mosquitoes, can be combined and work synergistically in a push-pull system. Push-pull systems have been shown to reduce house entry and outdoor biting rates of malaria vectors and so have the potential to control other outdoor biting mosquitoes such as Aedes aegypti that transmit arboviral diseases. In this study, semi-field experiments were conducted to evaluate whether a push-pull system could be used to reduce bites from Aedes mosquitoes.

Methods: The push and pull under investigation consisted of two freestanding transfluthrin passive emanators (FTPE) and a BG sentinel trap (BGS) respectively. The FTPE contained hessian strips treated with $5.25 \mathrm{~g}$ of transfluthrin active ingredient. The efficacies of FTPE and BGS alone and in combination were evaluated by human landing catch in a large semi-field system in Tanzania. We also investigated the protection of FTPE over six months. The data were analyzed using generalized linear mixed models with binomial distribution.

Results: Two FTPE had a protective efficacy (PE) of $61.2 \%$ (95\% confidence interval (Cl): 52.2-69.9\%) against the human landing of Ae. aegypti. The BGS did not significantly reduce mosquito landings; the PE was $2.1 \%$ (95\% Cl: $-2.9-7.2 \%)$. The push-pull provided a PE of $64.5 \%$ (95\% Cl: 59.1-69.9\%). However, there was no significant difference in the PE between the push-pull and the two FTPE against Ae. aegypti $(P=0.30)$. The FTPE offered significant protection against Ae. aegypti at month three, with a PE of $46.4 \%$ (95\% Cl: $41.1-51.8 \%)$, but not at six months with a PE of $2.2 \%$ (95\% Cl: $-9.0-14.0 \%)$.

Conclusions: The PE of the FTPE and the full push-pull are similar, indicative that bite prevention is primarily due to the activity of the FTPE. While these results are encouraging for the FTPE, further work is needed for a push-pull system to be recommended for Ae. aegypti control. The three-month protection against Ae. aegypti bites suggests that FTPE would be a useful additional control tool during dengue outbreaks, that does not require regular user compliance.
\end{abstract}

Keywords: Spatial repellent, Odor-baited trap, FTPE, Push-pull, BG-sentinel trap, Transfluthrin, Aedes aegypti

*Correspondence: mmohamed@ihi.or.tz

1 Environmental Health and Ecological Sciences, Ifakara Health Institute,

P.O. Box 74, Bagamoyo, Tanzania

Full list of author information is available at the end of the article

c) The Author(s) 2020. This article is licensed under a Creative Commons Attribution 4.0 International License, which permits use, sharing, adaptation, distribution and reproduction in any medium or format, as long as you give appropriate credit to the original author(s) and the source, provide a link to the Creative Commons licence, and indicate if changes were made. The images or other third party material in this article are included in the article's Creative Commons licence, unless indicated otherwise in a credit line to the material. If material is not included in the article's Creative Commons licence and your intended use is not permitted by statutory regulation or exceeds the permitted use, you will need to obtain permission directly from the copyright holder. To view a copy of this licence, visit http://creativeco mmons.org/licenses/by/4.0/. The Creative Commons Public Domain Dedication waiver (http://creativecommons.org/publicdomain/ zero/1.0/) applies to the data made available in this article, unless otherwise stated in a credit line to the data. 


\section{Background}

The Aedes aegypti mosquito is the primary vector of many arboviral diseases of public health importance, including dengue, yellow fever, Zika, and chikungunya [1-3]. The risk of contracting an arboviral disease is increasing as the world becomes more urbanized, because Ae. aegypti thrive in urban settings [4]. Dengue vector control is centered on larval source management, treatment of resting surfaces with insecticides and with space spraying as a response to disease outbreaks [5]. However, insecticides used for both space spraying and larviciding are short lasting and require a high frequency of reapplication to achieve sustained vector control. Larval habitat reduction is more sustainable but is not always practically or economically feasible in dengue-endemic countries.

Personal protection measures are also recommended during disease outbreaks through the use of appropriate clothing or topical repellents [6]. Topical repellents such as DEET (N, N-diethyl-m-toluamide) have demonstrated efficacy in reducing mosquito bites [7], and are also recommended for arbovirus prevention among military personnel and travelers [8]. However, there have been no studies to demonstrate their efficacy in reducing arboviral disease transmission. Topical repellents require frequent reapplication, which inevitably results in poor user compliance, and consequently coverage levels that are insufficient to interrupt disease transmission [9]. Each of the current control tools for Aedes has clear limitations and therefore, the development of complementary control tools to help fill these gaps is needed.

Spatial repellents $[10,11]$ and odor-baited traps [12, 13] have been suggested for the control of Aedes mosquitoes. Spatial repellents provide a bite-free space using repellent chemicals that passively evaporate at room temperature (emanators) or that are actively dispersed through heating coils, mats or vaporizers [14]. By removing the need for individual application, higher coverage levels may be possible with spatial repellents compared to topical repellents. Furthermore, spatial repellents may last for days, even months, after one application reducing the hassle of re-application and potentially increasing the protection even further through high effective coverage. Hessian strips treated with transfluthrin used as passive emanators have been shown to reduce human landing rates by $>90 \%$ for Culex and Anopheles mosquitoes in both semi-field and field experiments for up to six months $[11,15]$.

Odor baited traps, have been used extensively for mosquito monitoring and have recently been found to have public health benefits by reducing the population density of malaria and dengue vectors when deployed at a large scale in sufficient numbers to ultimately decrease disease transmission [13, 16, 17]. It has been shown that both spatial repellents and odor-baited traps used individually can be effective for the control of Ae. aegypti. These tools work by providing personal protection through reducing human-vector contact and also providing community protection by reducing the mosquito population size.

The push-pull control strategy originated from studies of agricultural pests showing that the practice of repelling "pushing" insects from one area and attracting "pulling" them to another, increases crop production [18]. The same strategy may be applied to control disease-transmitting mosquitoes of public health importance using spatial repellents and odor baited traps [19] and mathematical models have predicted that this control strategy may reduce entomological inoculation rate (EIR) by 20-fold for indoor-biting malaria-transmitting mosquitoes [19]. While push-pull control tools have also been tested against Aedes, successful laboratory results did not transfer to semi-field settings [14] with the researchers hypothesizing that the spatial repellent chemicals did not reach effective concentrations. In this study we investigate a new push-pull combination for Aedes using technologies that have individually proven successful under semi-field and field conditions. For the push component, a transfluthrin-treated hessian strip [15] was adapted to make a freestanding transfluthrin passive emanator (FTPE). The widely studied BGS was selected as the pull component of the system. Here, we investigate the efficacy of push and pull individually and then in a combination as push-pull to see if the combination provided better efficacy measured as a reduction in mosquito landings than either of the individual components. We also measured the duration of protective efficacy of the FTPE over a six-month period.

\section{Methods \\ Study design}

This study investigated the efficacy of the FTPE and BGS in a push-pull system to reduce human-landing rates compared to the control (no intervention). We also determined if the combination of FTPE and BGS was better than either FTPE or BGS alone whereby the following treatment arms were compared: (i) two FTPE vs negative control; (ii) BGS trap vs negative control; and (iii) the combination of FTPE and BGS vs negative control. The study design was a randomised block design over 16 days per treatment arm. Each intervention and its control were assigned to one of two separate compartments in the semi-field system (SFS) for a block of 4 days, after which the treatment and its control were switched between compartments. Preliminary experiments showed that removing FTPE immediately after experiment and airing the compartment for $20 \mathrm{~h}$ 
was enough to prevent any residual effect of the transfluthrin. In each block of 4 days, 4 volunteers rotated daily between chambers.

\section{Study site}

The experiment was conducted in the SFS located in Bagamoyo, Tanzania, between January 2018 and December 2018. The SFS measures $21 \times 29 \times 4.5 \mathrm{~m}$ with two compartments $(21 \times 9 \mathrm{~m})$, separated by a corridor. A heavy-duty polyethene wall separates these compartments preventing air movement between the chambers and reducing any chance of cross-contamination when working with spatial repellents or other aerosols. The SFS allows for controlled experiments with set densities of disease-free mosquitoes to be conducted under field-like climatic conditions throughout the year [20].

\section{Mosquitoes}

Laboratory reared, pyrethroid susceptible Ae. aegypti (Bagamoyo strain) were used. Susceptibility bioassays performed prior the implementation of the experiment following the World Health Organization (WHO) guidelines [21] showed that mortality of these mosquitoes was $>99 \%$ after exposure to all the pyrethroid insecticides tested (deltamethrin $(0.03 \%)$, permethrin $(0.25 \%)$ and alpha-cypermethrin (0.03\%). These mosquitoes were colonized from Bagamoyo in December 2015. Larvae were fed on Tetramin ${ }^{\circledR}$ fish food and adult mosquitoes on $10 \%$ sucrose ad libitum, and cow blood meals (heparinized) were given to adult females for egg production using a membrane-feeding assay. The colony was maintained at $27 \pm 5{ }^{\circ} \mathrm{C}$ and $50-99 \%$ relative humidity.

For this experiment, 3-8 day-old female mosquitoes, previously unfed with blood were used. These mosquitoes were sugar-starved for $12 \mathrm{~h}$ before the start of experiments. Female mosquitoes that were responsive to human odour were selected from three different rearing cages and transferred to small releasing cages. Selection from cages was done by placing a hand close to the cage and choosing only aggressive host-seeking mosquitoes.

\section{Preparation of the freestanding transfluthrin passive emanator (FTPE)}

We designed a device that can easily be placed anywhere in the peri-domestic space (Fig. 1a-e). The emanator passively releases transfluthrin vapors into the surrounding area through evaporation. The device is a stool-like structure that supports hessian strips (made from plants of the species Corchorus olitorius or C. capsularis also called jute, burlap or gunny sacks), treated with the emulsifiable concentrate (EC) transfluthrin active ingredient (Bayothrin EC; Bayer AG Monheim am Rhein, Germany) as the push. The hessian fabrics were chosen as they have been shown to retain transfluthrin for up to 6 months due to their high cellulose content $[11,15]$. The hessian fabric was locally bought, washed with $\mathrm{OMO}^{\circledR}$ detergent powder (Unilever Kenya Limited, Nairobi, Kenya) and dried under direct sunlight. The fabrics were cut into strips with a surface area of $0.5 \mathrm{~m}^{2}(10 \mathrm{~cm} \times 5 \mathrm{~m})$ and treated with $5.25 \mathrm{~g}$ of emulsified concentrate transfluthrin and left to dry under the shade in the SFS (Fig. 1b, c) to prevent photolysis of transfluthrin. The strips were then wound around a pole into a spiral and sealed with outer wire mesh to prevent access to the treated hessian ribbon by children or animals (Fig. 1d). Two FTPE with a total of $10.50 \mathrm{~g}$ (5.25 g each) of transfluthrin were used per experiment.

\section{BG sentinel trap}

The BGS (Biogents AG, Regensburg, Germany) has been widely used as the standard trap for collection of adult Aedes mosquitoes [22, 23]. The BGS was used with a Biogents-Lure (BGL) and carbon dioxide as a pull. The BGL is a synthetic lure consisting of lactic acid, caproic acid and ammonium bicarbonate dispensed via granules [23]. It is effective for 5 months; however, for the purpose of this experiment a new lure was used for each experimental round of sixteen days. Carbon dioxide was released from a pressurized cylinder at the rate of $500 \mathrm{ml} / \mathrm{min}$, using an acrylic gas flow meter (Hangzhou Darhor, Technology Co. Limited, Zhejiang, China).

\section{Procedure to determine the protective efficacy of the FTPE and odor baited trap}

To simulate the peridomestic setting, human landing catches (HLC) were performed with a volunteer sitting $2 \mathrm{~m}$ from an experimental hut inside the SFS (Fig. 2a-c). For the "push" alone evaluation, two FTPE were positioned $6 \mathrm{~m}$ apart with the human volunteer sitting in between them conducting the HLC (Fig. 2a). During the "pull" alone evaluation, the BG-sentinel was placed $10 \mathrm{~m}$ away from the HLC (Fig. 2b) as this exceeds the maximum distance over which mosquitoes select between hosts so that the action of the trap could be measured independently of the effect of the human collector [24]. For the push-pull evaluation, both FTPE and the BGS were used and positioned as described above in the push and pull setups (Fig. 2c). In the control, untreated emanators and HLC were used.

The FTPE were positioned in the experimental chambers $45 \mathrm{~min}$ before the experiment started to allow the release of active ingredients into the experimental space, 
and mosquitoes were transferred to the buffer chamber (corridor) of the SFS $1 \mathrm{~h}$ before the experiment began to allow for acclimatization. During the acclimatization process, mosquitoes remained free from transfluthrin exposure. After the acclimatization period, cages with approximately 25 mosquitoes each were positioned in the 4 corners of both compartments (approximately 100 mosquitoes per compartment/treatment). Mosquitoes were released at $07: 00 \mathrm{~h}$ by a gentle pull of the strings connecting the releasing cages and the chair where volunteers were sitting. The experiment was conducted from $07: 00 \mathrm{~h}$ to $10: 00 \mathrm{~h}$ to reflect natural biting time for Aedes mosquitoes [25].

The volunteers conducted HLC, collecting mosquitoes that landed on the area between the ankle and the knee for 3 consecutive hours. All volunteers were males aged between 25-40. They were non-drinkers, non-smokers and were asked not to apply perfume or bathe using perfumed soap, before the experiments. During the experiment, volunteers wore shorts, covered shoes, and bug jackets to standardize the area available for mosquito landings. Mosquitoes were recaptured continuously for 50 min using a mouth aspirator. After 50 min the volunteers would take a break for $10 \mathrm{~min}$, after which a new paper cup labeled with the time and date were used. Collected mosquitoes were transferred to the insectary for sorting. After the experiment, mosquitoes that were not collected during the HLC were recaptured using locally made Prokopack aspirators and killed by freezing to prepare the SFS for the next day's experiment. A Tinytag ${ }^{\circledR}$
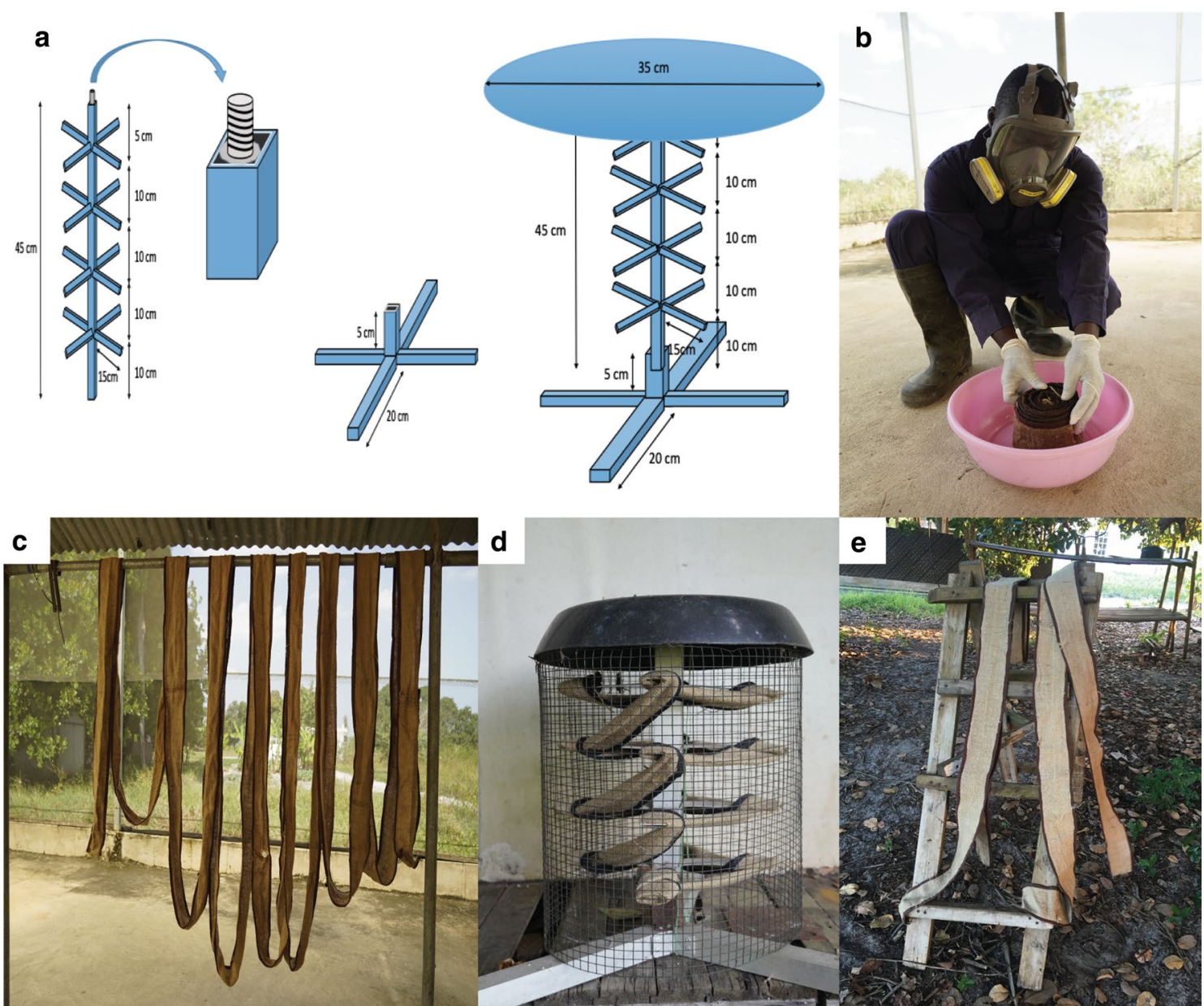

d
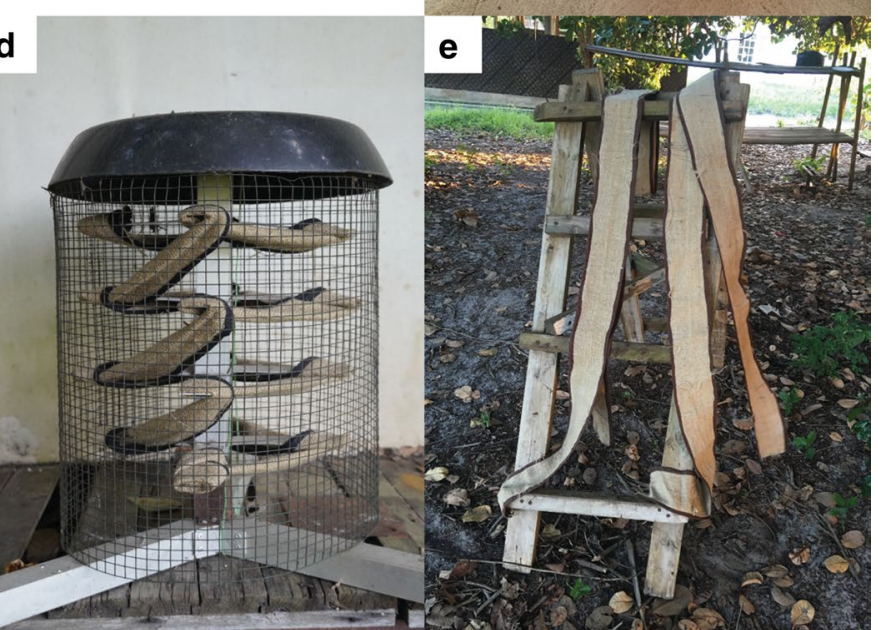

Fig. 1 Preparation of the freestanding emanator (FTPE) "push". a Design of the freestanding emanator. The device measures $50 \mathrm{~cm}$ in height and $40 \mathrm{~cm}$ in diameter. It consists of three parts; the top cover, the central square pipe and a base. The central pipe rests on the base that supports the device. The pipe is divided into four portions $10 \mathrm{~cm}$ apart where small branches of an aluminium flat bar $15 \mathrm{~cm}$ long are attached. $\mathbf{b}, \mathbf{c}$ Transfluthrin impregnation and drying of the hessian strips under the shade. $\mathbf{d}$ The FTPE (the hessian strip enclosed with the wire mesh). e The transfluthrin-treated hessian strips placed under the shade between the experiment for "field aging" for the duration of efficacy experiment 
view 2 data logger (model TV-4500; Gemini data logger, Chichester, UK) was placed inside the SFS throughout the experiment to record temperature and relative humidity.

\section{Experiment to assess the longevity of the FTPE}

To assess the longevity of FTPE protection, the devices were evaluated at 0,3 and 6 -months post-impregnation. The same set up as described previously (Fig. 2a) was followed. Between the evaluations, the emanators were "field aged" by storing the hessian strips in an outdoor environment under a tree in the shade to simulate aging on a verandah of a house, i.e. placed outdoors under ambient conditions, protected from direct sunlight and rain (Fig. 1e).

\section{Sample size}

Sample size calculations were performed using simulation-based power analysis [26] in R statistical software version 3.02 (http://www.r-project.org) with a significance level of 0.05 for rejecting the null hypothesis. Data analysis for experimental data was conducted using generalized linear mixed models (GLMMs). Therefore, one thousand simulations of generalized linear mixed models approximating those that will be used to analyze project data were run using the same experimental design. The power to predict the difference in mosquito landings between control and treatment was estimated as the proportion of the 1000 simulated data sets in which the null hypothesis was rejected when the generalized linear mixed model was run. Parameters were set at $10 \%$ estimated variability between chambers, $10 \%$ variability between mosquito releases and $10 \%$ variability between volunteers. Simulations indicated that with an estimated 100 mosquitoes released per day and 60\% recapture of released mosquitoes in the control, there was $94 \%$ power (95\% CI: $92-96 \%$ ) to detect a 50\% reduction in mosquito landings in the treatment arm after 16 nights of experimentation. Furthermore, there was $70 \%$ power (95\% CI: 68-72\%) to detect a $15 \%$ difference between the treatments.

\section{Data analyses}

Data were entered in Microsoft Excel 2010 and analyzed in Stata 13 (StataCorp, College Station, TX, USA). The data were analyzed to determine the efficacy of each

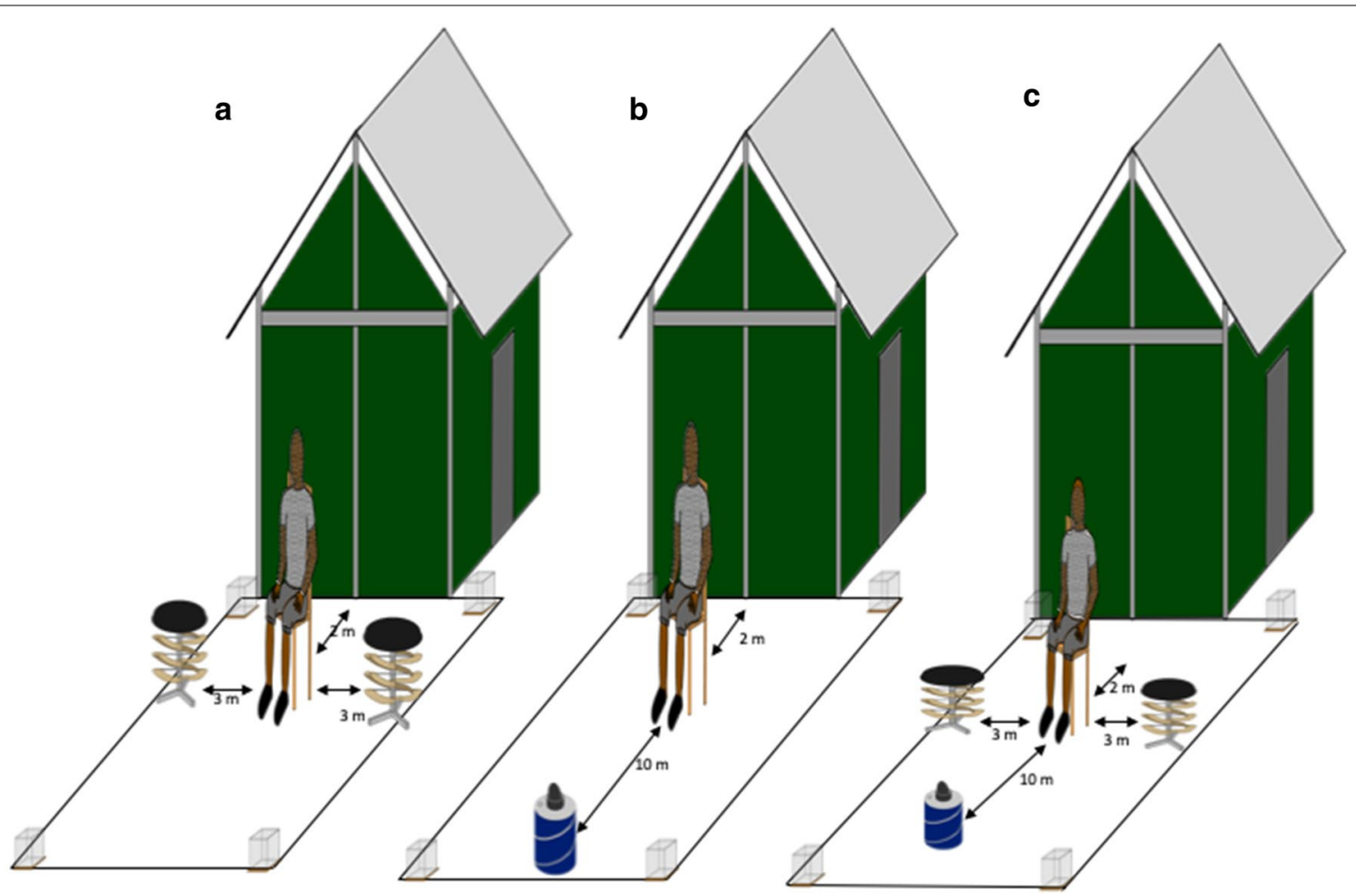

Fig. 2 Schematic representation for the experiment in the SFS. a The arrangement of push intervention. $\mathbf{b}$ The BGS positioned $10 \mathrm{~m}$ away from the human volunteer during the pull alone evaluation. c The positions of interventions during the push-pull evaluation. In each setup, a human volunteer preforming HLC sat $2 \mathrm{~m}$ away from the experimental hut and if push was involved, two FTPE were positioned $3 \mathrm{~m}$ on each side of the HLC volunteer. Small boxes at the corner represent the releasing cages positioned where mosquitoes were released 
intervention (push alone, pull alone and push-pull) to reduce the human landing rate compared to the control. The mean percentage recapture and confidence intervals were calculated for each intervention and control. From this daily protective efficacy was measured by comparing the human landing rate on a volunteer, with the intervention to the negative control using the following formula and the overall arithmetic mean PE and 95\% confidence interval for the experiment was calculated:

$$
\text { Protective efficacy }(\mathrm{PE})=[(\mathrm{C}-\mathrm{T}) / \mathrm{C}] \times 100 \%
$$

where $\mathrm{C}$ stands for the number of mosquitoes landing in the control and $\mathrm{T}$ is the number of mosquitoes landing in the treatment.

The effect of each intervention was determined by fitting a GLMM with a binomial distribution and logit function. The binomial distribution was chosen, as the dependent variable was the proportion of recaptured mosquitoes out of those released. Independent fixed effect categorical variables were treatment (intervention or control), compartment, volunteer, block (push, pull, push-pull) and an interaction term between treatment and block. Day was included as a random effect.

To determine the longevity of FTPE across 6 months after impregnation; a GLMM with a binomial distribution and logit function was also used. For this model, the dependent variable was again the proportion of recaptured mosquitoes. Independent fixed effect categorical variables were treatment (intervention or control), compartment, volunteer, month of testing (month 0 , month 3 , month 6) and an interaction term between treatment and months. This interaction was used to determine if the protective efficacy of FTPE changed between months. Day was included as a random effect.

During the evaluation, there was no significant association between humidity and temperature on the proportion of recaptured mosquitoes $P=0.705$ and $P=0.203$ for the humidity and temperature, respectively. Therefore, these variables were not included in the GLMM.

\section{Results}

Protective efficacy of the push-alone, pull-alone and push-pull

During the 16 experiment days, 439/1600 (27\%) of released mosquitoes were captured in the presence of FTPE, 926/1600 (58\%) in the presence of BGS and 349/1600 (22\%) in the presence of push-pull, whereas in the control compartment, 951-1114/1600 (59-71\%) of released mosquitoes were recaptured (Table 1). The average temperature during the experiments was $25.4{ }^{\circ} \mathrm{C}\left(21.0-26.0{ }^{\circ} \mathrm{C}\right)$ and the average relative humidity was $90 \%$ (68-100\%). The full dataset is available in Additional file 1: Dataset S1.
The protective efficacy (PE) of FTPE against Ae. aegypti bites was $61.0 \%$ (95\% CI: 52.2-69.9\%; odds ratio (OR): 0.14, 95\% CI: 0.12-0.16, $P<0.0001$ ) (Fig. 3, Table 1). The BGS did not reduce Ae. aegypti landings on a human volunteer sitting $10 \mathrm{~m}$ away with PE of 2.1\% (95\% CI: -2.97.2\%; OR: 0.92, 95\% CI: 0.81-1.08, $P=0.371$ ) (Fig. 3). The combination of FTPE and the BGS significantly reduced Ae. aegypti landings with PE of $64.5 \%$ (95\% CI: 59.1-69.9\%; OR: 0.16, 95\% CI: 0.14-0.19, $P<0.0001)$ (Fig. 3, Table 1). The proportion of mosquitoes caught by BGS used alone 6.1\% (95\% CI: 5.1-6.1\%) or in combination with FTPE 6.1\% (95\% CI: 5.0-7.3\%) showed no significant difference $(P=0.34)$. This indicates that the push and pull components were not working synergistically, with a majority of protection provided by the push alone.

\section{Comparing the performance of the push-alone, pull-alone and push-pull}

A significant interaction between type of intervention (push, pull or push-pull) and treatment in the model confirmed that the majority of protective efficacy in the push-pull was provided by the FTPE. The protective efficacy of push-alone, as described above, was significantly greater than pull-alone $(P<0.0001)$ while the protective efficacy of push-alone was not significantly different than the full push-pull system $(P=0.29)$. There was no significant difference in the compartment $(P=0.29)$ or volunteers $(P>0.05$ for all volunteers $)$ on the number of recaptured mosquitoes.

\section{Protective efficacy of the push-alone over six months}

There was a significant interaction between month and treatment showing that the PE of the FTPE decreased over time $(P<0.0001)$. At 3 months after impregnation, the FTPE was still providing significant protection against Ae. aegypti with a PE of $46.4 \%$ (95\% CI: 41.1-51.8\%; OR: 0.26, 95\% CI: $0.22-0.29, P<0.0001$ ). However, when the FTPE were tested at month 6 after impregnation, no significant protection was offered (OR: $0.91,95 \% \mathrm{CI}: 0.79-1.05, P=0.22$ ), with protective efficacy dropping to $2.2 \%$ (95\% CI: $-9.0-14.0$ ) (Fig. 4).

\section{Discussion}

The current vector control tools used against Aedes mosquitoes have several limitations, necessitating the development and testing of additional tools for proactive dengue prevention. This study demonstrated that while the push-pull system reduced human-vector contact of Ae. aegypti mosquitoes, the majority of protection was provided by the FTPE. The likely reason for this is the poor response of mosquitoes to the BGS in the presence of humans. Numerous previous studies on push-pull 
technologies have demonstrated the higher efficacy of the push than the pull [14, 27-29]. While the push-pull system may need further development, the success of the FTPE was encouraging and indicated their potential for the control of arboviral diseases.

The FTPE remained protective for three months, therefore, it is possible to meet high levels of coverage in an urban setting with just one application, reducing the difficulties of re-application and user non-compliance. These promising results indicate that FTPE could potentially be used to protect individuals in the peridomestic space longer than current personal protection methods [5]. This may be particularly useful during arboviral disease outbreaks that tend to coincide with the 3-5-month rainy season [30].

Our finding of FTPE efficacy is consistent with previous studies evaluating transfluthrin against Anopheles arabiensis and Ae. aegypti mosquitoes [31-33]. However, we may not generalize that these transfluthrintreated passive emanators provide protection in all geographical locations. It is important to consider environmental factors and susceptibility status of the mosquitoes before implementing any control strategy. For example, in a windy environment, the active ingredient can be blown away, therefore reducing the concentration needed in the air to repel mosquitoes. Temperature can affect the vaporization of transfluthrin and

Table 1 The percentage of mosquito landings, protective efficacy and the odds ratio for each intervention

\begin{tabular}{llllll}
\hline Experiment & Treatment & Control & PE $(95 \% \mathrm{Cl})$ & OR (95\% Cl) & $P$-value \\
\hline Push & $439 / 1600(27 \%)$ & $1141 / 1600(71 \%)$ & $61.2 \%(52.2-69.9)$ & $0.14(0.12-0.16)$ & $<0.0001$ \\
Pull & $926 / 1600(58 \%)$ & $951 / 1600(59 \%)$ & $2.1 \%(-2.9-7.2)$ & $0.92(0.81-1.08)$ & 0.371 \\
Push pull & $349 / 1600(22 \%)$ & $999 / 1600(62 \%)$ & $64.5 \%(59.1-69.9)$ & $0.16(0.14-0.19)$ & $<0.0001$ \\
\hline
\end{tabular}

Notes: Numbers in the control and treatment groups refer to the total number of mosquitoes caught/released during each experiment and the percentage recaptured are in parentheses. Also shown are the estimates for PE and $95 \% \mathrm{CI}$. Finally, the OR and $P$-value derived from the GLMM model are presented

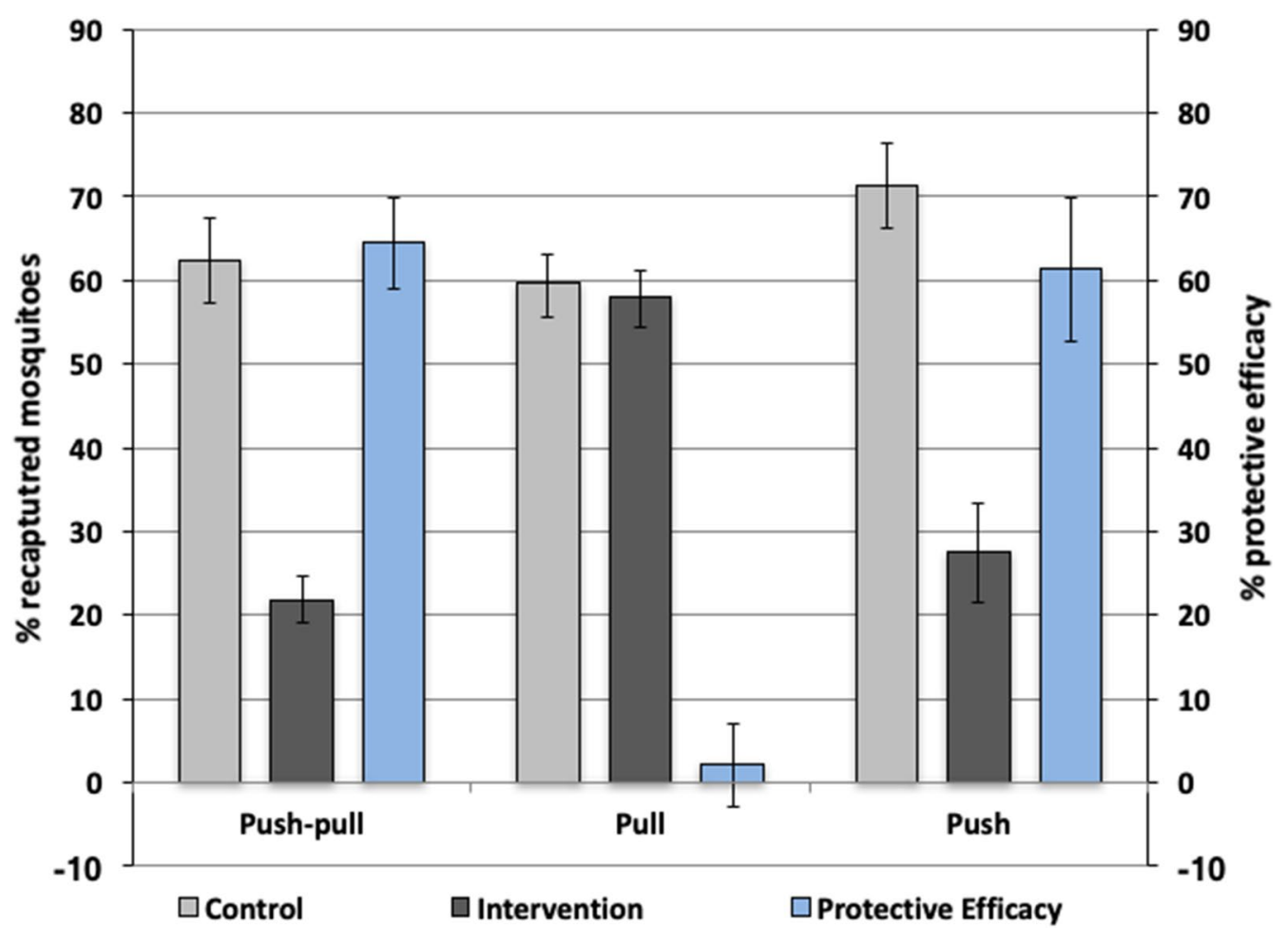

Fig. 3 Percentage of recaptured mosquitoes and protective efficacy. The arithmetic mean percentage of mosquitoes recaptured by HLC in the presence of the BGS (pull), FTPE (push), spatial repellent emanator and odour-baited trap (push-pull) compared to the control. The secondary axis shows the $\%$ protective efficacy of each intervention. Error bars represent the $95 \%$ confidence intervals 
thereby its concentration and protective efficacy. It has been reported that the optimal temperature for a transfluthrin-treated emanator to provide maximum protection ranges between $21-30{ }^{\circ} \mathrm{C}$, with a reduction in protection specifically in lower temperatures [11]. This suggests that in geographical locations where the daytime ambient temperature is below $21^{\circ} \mathrm{C}$ the efficacy of these emanators for prevention of Ae. aegypti bites may be impaired. However, these experiments were conducted within the temperature range of $20.9-25.5{ }^{\circ} \mathrm{C}$, which is optimal for transfluthrin evaporation. Also, it was reported that resistance to transfluthrin could be selected for among laboratory Ae. aegypti which were less sensitive to transfluthrin afterward [34]. This implies that the efficacy of these emanators may be impaired in the area with confirmed pyrethroid resistant Aedes mosquitoes.

In this study, we have demonstrated that the BGS positioned 10 meters away did not significantly protect a person from mosquito bites. However, previous experiments have shown that the BGS used alone is an effective trap for sampling Ae. aegypti [17, 23, 35]. In this experiment, the BGS was placed near a human volunteer and they were the only "hosts" available. This demonstrated that the human cues were significantly more attractive to Aedes than the cues from the BGS. Preliminary work in the semi-field system indicated that the BGS caught many Aedes in the absence of the human volunteers, revealing that the efficacy of BGS is relative to the proximity and density of humans. This has also been observed in other studies with humans outcompeting traps at short range [12] and that whole human odor is optimally attractive to anthropophagic mosquitoes [36]. While the BGS did not provide personal protection by reducing human-vector contact as the removal trap, it could still provide some level of community protection if used on a larger scale, although other traps such as the autocidal gravid trap may be more feasible for removal trapping [37] as they do not require carbon dioxide.

The number of mosquitoes successfully caught by the BGS during the push-pull or the pull only configuration was the same. While this showed that transfluthrin did not actively push mosquitoes into the trap it also indicated that transfluthrin exposure outdoors does not inhibit mosquitoes entering the BGS. This is contrary to Salazar et al. [38] who reported that exposing mosquitoes to transfluthrin significantly lowered trap catches. However, trap catches were not affected if the mosquitoes were allowed to recover for $12 \mathrm{~h}$ before BGS trap evaluation [38]. This suggests that the mode of action of transfluthrin is dose- and distancedependent and repellency is reversible (i.e. mosquitoes are

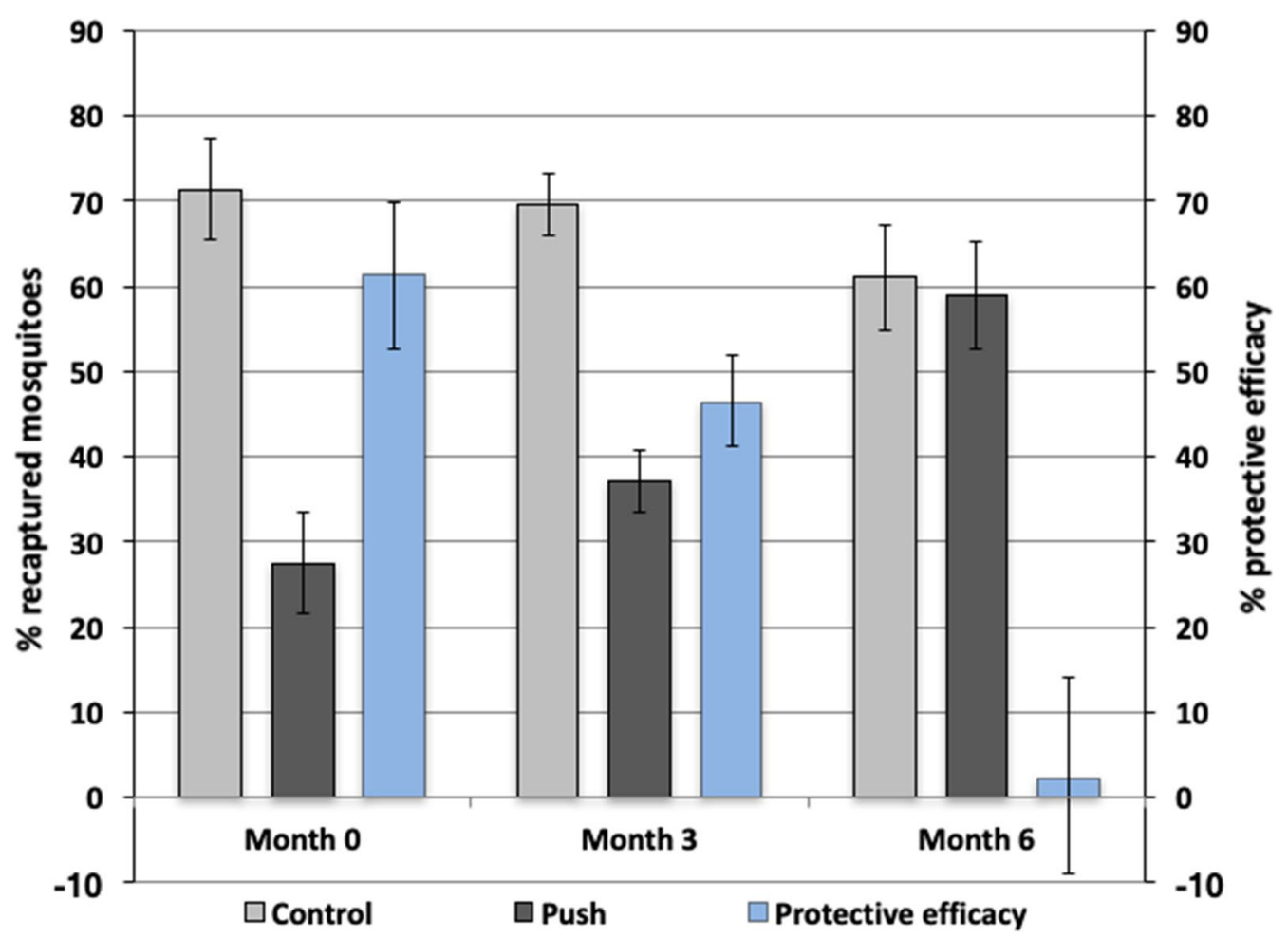

Fig. 4 The duration of efficacy of the FTPE. The arithmetic mean percentage of mosquitoes recaptured by HLC in the compartment with FTPE compared to the control up to six months after treatment. The secondary axis represents the \% protective efficacy of the push at each time point. Error bars represent the $95 \%$ confidence intervals 
disarmed for a period). Use of a higher dose could be further optimized to increase the kill of mosquitoes or cause a prolonged state where mosquitoes are disarmed and unable to find a host. This could prevent diversion of repelled mosquitoes from repellent users to non-users in a community [39].

We have shown that FTPE remain protective for three months following impregnation. This is a relatively short duration compared to the previous studies conducted against malaria vectors demonstrating that transfluthrintreated strips remain protective for up to six months against Anopheles mosquitoes [11, 15]. A possible explanation for these differences could be due to the variation in transfluthrin dosage, mosquito species and the distance from the emanator where HLC was performed. Aedes aegypti is highly anthropophilic and requires higher doses of topical repellents to be repelled than other species with more diverse host preferences such as $A n$. arabiensis [40]. In the present study, $10.5 \mathrm{~g}$ of transfluthrin $(5.25 \mathrm{~g}$ on each of two FTPE) was used against Ae. aegypti and HLC conducted three meters from the emanators, whereas in the study by Ogoma et al. [11, 15] the volunteer sat one meter from a strip enclosing them on all four sides at an application of $15.1 \mathrm{~g}$ transfluthrin against An. arabiensis mosquitoes. Ogoma et al. showed that hessian treated with $1.51 \mathrm{~g}$ of transfluthrin resulted in air concentration $>1000$ times lower than the maximum acceptable concentration for long term inhalation exposure of a human being as defined by the regulatory authorities of the European Union (EU) [11]. In general, the efficacy of the emanators in both studies decreases over time as the result of the loss of transfluthrin due to evaporation. To ensure long-term efficacy of the FTPE, a thicker layer of the hessian strips could be used or transfluthrin doses increased, provided they remain within the margin of safety for chronic inhalation exposure [41]. The FTPE is a simple proof of concept prototype and further work is required to develop a product for use as a public health intervention, including standardizing the release rate of the transfluthrin through standardization of the material upon which the transfluthrin is applied, and improvement of the delivery unit to ensure it is cost-effective and tamperproof. This may also include the application of UV protection to the transfluthrin-treated material to prolong its efficacy outdoors [42].

The use of FTPE improves user compliance, as they are portable and easy to use which facilitates round the clock protection at the desired location in the peridomestic area. The replacement of FTPE after every three months avoids the problems associated with personal topical repellents that require daily application, but tend to be applied only when people notice mosquito bites [43, 44], resulting in lack of public health benefit [45]. As the device potentially provides protection to multiple people without the need for personal reapplication, it is likely a convenient approach to bite prevention outside of sleeping hours and to be more acceptable among community members for protection of the whole family [46]. Therefore, the FTPE are suitable for targeted distribution among high-risk populations such as those reported to harbor Aedes breeding sites during the outbreak [47]. Finally, the FTPE is a passive device that does not produce smoke (like mosquito coils), which improves user acceptance and reduces potential exposure to organic pollutants.

Dengue tends to be focal in transmission with Aedes spp. commonly having a short flight range, although there are exceptions [48]. Therefore, transmission is primarily mediated between locations by movement of infected individuals [49]. These devices are portable and may be deployed anywhere; they could be very useful if provided to those households with confirmed dengue, deployed in entrance points (port and airport) where travelers are coming in from other countries, or in places where new cases are suspected or an outbreak is reported, such as markets [1]. The high mosquito toxicity of transfluthrin is an important feature of this tool, as it has the potential to kill a substantial proportion of mosquitoes that encounter the insecticide and reduce vector densities and vectoral capacity. Further work into the impact of such devices on the resistant Ae. aegypti and mortality of free-flying mosquitoes is recommended.

There were two limitations of this study, including the use of laboratory-reared mosquitoes that may exhibit different behaviors to wild mosquitoes. Also, the data for the longevity experiment were collected at 0,3 and 6 months only. Whereas significant protective efficacy (44\%) was observed up to three months after impregnation the FTPE. With this experimental design we missed the exact time point (between 3 and 6 months) when the FTPE stopped providing significant protection. We recommend that future studies conducting the same kind of experiment need to conduct weekly or monthly evaluations in order to provide a more precise estimate of efficacy over time, especially when testing the label claims of long-lasting spatial repellent products. Moreover, we only evaluated one distance setup for use of the "pushpull" in the SFS. In the field, the positioning of this system will vary due to the local building layout resulting in varying protection levels. We recommend that further studies on push-pull should (i) focus on improving the attraction of pull components, since they need to outcompete humans, and (ii) explore optimal positioning of components to determine the effective distance at which the push and pull could work synergistically.

\section{Conclusions}

In this study, we have demonstrated that FTPE has potential to reduce bites from Ae. aegypti mosquitoes for up to three months. Using a combination of passively emanated 
transfluthrin and BGS as a push-pull did not provide any additional protection, with the majority of the protection originating from the push component. Additional work is needed in the field and through mathematical modeling to determine if the number of mosquitoes caught in the BGS would provide additional community protection when added to the FTPE. The FTPE are portable and easy to use which facilitates round the clock protection at the desired location in the peridomestic area for much longer than most currently available personal protection methods.

\section{Supplementary information}

Supplementary information accompanies this paper at https://doi. org/10.1186/s13071-020-04263-3.

Additional file 1: Dataset S1. The dataset from the semi-field evaluation of the push pull for the control of outdoor biting mosquitoes that support the conclusions of this article.

\section{Abbreviations}

FTPE: Freestanding transfluthrin passive emanators; Cl: Confidence interval; DEET: N,N-diethyl-meta-toluamide; BGS: Biogents sentinel trap; BGL: Biogents lure; EIR: Entomological inoculation rate; EC: Emulsifiable concentrate; SFS: Semi field system; HLC: Human landing catches; GLMM: Generalized linear mixed model; PE: Protective efficacy; UV: Ultraviolet; IHI: Ifakara Health Institute; IRB: Institute Review Board; NIMR: National Medical Research Institute; WHO: World Health Organization.

\section{Acknowledgements}

We would like to thank Athuman Kambagha and Frank Tenywa for establishment of the Ae. aegypti mosquito colony from the field. Thanks to Amanda Ross for advice on statistical analysis. Bayer AG generously donated transfluthrin used in the experiment. We also thank the 2 reviewers of this manuscript for their constructive inputs.

\section{Authors' contributions}

MMT, AS and SJM conceived and designed the study. MMT and HC supervised the semi-field experiment, volunteers and data collection. MT AS, JKS and SJM performed data analysis. MT drafted the manuscript. AS, CS and SJM revised the manuscript. CS drew the schematic representation of the semi-field system experiment. MMT and JDM designed and drew the FTPE. All authors read and approved the final manuscript.

\section{Funding}

Ifakara Health Institute covered experimental costs. Salaries for MMT, SM and AS were funded through a grant from the Innovative Vector Control Consortium (IVCC). IVCC would like to acknowledge that source funding for the'PushPull' project came from the Bill \& Melinda Gates Foundation and UKaid.

\section{Availability of data and materials}

All data generated or analysed during this study are included in this published article and its additional file.

\section{Ethics approval and consent to participate}

The volunteers that participated in this experiment were IHI employees, fully trained and skilled in performing HLC. They were recruited voluntarily through written informed consent, after the risks and benefits of the study procedures and their right to leave at any time during the study was clearly explained. All the mosquitoes used in this experiment were laboratory-reared and free from arboviral diseases. The study was approved by the IHI Institute Review Board (IHI-IRB) and the National Institute for Medical Research, Tanzania (NIMR) with certificate number IHI/IRB/No: 024-2016 and NIMR/HQ/R.8a/Nol.IX/2381, respectively. Permission to publish this work was granted from the Director General of NIMR.

\section{Consent for publication}

Not applicable.

\section{Competing interests}

The authors declare that they have no competing interests. SJM, JKS and CS conduct contract product evaluation of a number of vector control tools.

\section{Author details}

${ }^{1}$ Environmental Health and Ecological Sciences, Ifakara Health Institute, P.O. Box 74, Bagamoyo, Tanzania. ${ }^{2}$ Swiss Tropical \& Public Health Institute, Socinstrasse 57, 4051 Basel, Switzerland. ${ }^{3}$ University of Basel, Petersplatz 1, 4001 Basel, Switzerland.

Received: 28 April 2020 Accepted: 23 July 2020

Published online: 31 July 2020

\section{References}

1. Mboera LE, Mweya CN, Rumisha SF, Tungu PK, Stanley G, Makange MR, et al. The risk of dengue virus transmission in Dar es Salaam, Tanzania during an Epidemic Period of 2014. PLoS Negl Trop Dis. 2016;10:e0004313.

2. Gould LH, Osman MS, Farnon EC, Griffith KS, Godsey MS, Karch S, et al. An outbreak of yellow fever with concurrent chikungunya virus transmission in South Kordofan, Sudan, 2005. Trans R Soc Trop Med Hyg. 2008;102:1247-54.

3. Wikan N, Smith DR. Zika virus: history of a newly emerging arbovirus. Lancet Infect Dis. 2016:16:e119-26.

4. Messina JP, Brady OJ, Golding N, Kraemer MUG, Wint GRW, Ray SE, et al. The current and future global distribution and population at risk of dengue. Nat Microbiol. 2019:4:1508-15.

5. Roiz D, Wilson AL, Scott TW, Fonseca DM, Jourdain F, Muller P, et al. Integrated Aedes management for the control of Aedes-borne diseases. PLoS Negl Trop Dis. 2018;12:e0006845.

6. WHO. Guideline for malaria vector control. Geneva: World Health Organization; 2019.

7. Lupi E, Hatz C, Schlagenhauf P. The efficacy of repellents against Aedes, Anopheles, Culex and Ixodes spp. - a literature review. Travel Med Infect Dis. 2013;11:374-411.

8. Fabrice L, Thierry A, Eric C, Marie-Laure D, Jean D, et al. Personal protection against biting insects and ticks. Parasite. 2011;18:93-111.

9. Gryseels C, Sambunny UK, Sluydts V, Durnez L, Phoeuk P, Suon S, et al. Factors influencing the use of topical repellents: implications for the effectiveness of malaria elimination strategies. Sci Rep. 2015;5:16847.

10. Achee NL, Michael JB, Robert F, Kileen GF, Steve L, James GL, et al. Spatial repellents: from discovery and development to evidence-based validation. Malar J. 2012;11:164.

11. Ogoma SB, Mmando AS, Swai JK, Horstmann S, Malone D, Killeen GF, et al. A low technology emanator treated with the volatile pyrethroid transfluthrin confers long term protection against outdoor biting vectors of lymphatic filariasis, arboviruses and malaria. PLoS Negl Trop Dis. 2017;11:e0005455.

12. Okumu FO, Killeen GF, Ogoma S, Biswaro L, Smallegange RC, Mbeyela E, et al. Development and field evaluation of a synthetic mosquito lure that is more attractive than humans. PLoS ONE. 2010;5:e8951.

13. Degener CM, Eiras AE, Azara TM, Roque RA, Rosner S, Nobre AA, et al. Evaluation of the effectiveness of mass trapping with BG-sentinel traps for dengue vector control: a cluster randomized controlled trial in Manaus, Brazil. J Med Entomol. 2014;51:408-20.

14. Obermayr U, Ruther J, Bernier UR, Rose A, Geier M. Evaluation of a pushpull approach for Aedes aegypti (L.) using a novel dispensing system for spatial repellents in the laboratory and in a semi-field environment. PLoS ONE. 2015;10:e0129878.

15. Ogoma SB, Ngonyani H, Simfukwe ET, Mseka A, Moore SJ, Killeen GF, et al. Spatial repellency of transfluthrin-treated hessian strips against laboratory-reared Anopheles arabiensis mosquitoes in a semi-field tunnel cage. Parasit Vectors. 2012;5:54.

16. Homan T, Hiscox A, Mweresa CK, Masiga D, Mukabana WR, Oria P, et al. The effect of mass mosquito trapping on malaria transmission and disease burden (SolarMal): a stepped-wedge cluster-randomised trial. Lancet. 2016;388:1193-201. 
17. Rafael MF, Avaro LE, Ricardo LO. Field evaluation of effectiveness of the BG-Sentinel, a new trap for capturing adult Aedes aegypti (Diptera: Culicidae). Mem Inst Oswaldo Cruz. 2006;101:321-5.

18. Cook SM, Khan ZR, Pickett JA. The use of push-pull strategies in integrated pest management. Annu Rev Entomol. 2007;52:375-400.

19. Menger DJ, Omusula P, Holdinga M, Homan T, Carreira AS, Vandendaele P, et al. Field evaluation of a push-pull system to reduce malaria transmission. PLOS ONE. 2015;10:e0123415.

20. Ferguson HM, Ng'habi KR, Walder T, Kadungula D, Moore SJ, Lyimo I, et al. Establishment of a large semi-field system for experimental study of African malaria vector ecology and control in Tanzania. Malar J. 2008;7:158.

21. WHO. Test procedures for insecticide resistance monitoring in malaria vector mosquitoes. Geneva: Word Health Organization, 2016. https:// www.who.int/malaria/publications/atoz/9789241511575/en/. Accessed 19 Apr 2020.

22. Schoeler GB, Schleich SS, Manweiler S, Sifuentes VL. Evaluation of surveillance devices for monitoring Aedes aegypti in an urban area of northeastern Peru. J Am Mosq Control Assoc. 2004;20:6-11.

23. Kröckel U, Rose A, Eiras ÁE, Geier M. New tools for surveillance of adult yellow fever mosquitoes: comparison of trap catches with human landing rates in an urban environment. J Am Mosq Control Assoc. 2006;22:229-38.

24. Gillies MT, Wilkes TJ. The range of attraction of single baits for some West African mosquitoes. Bull Ent Res. 1970;69:225-35.

25. Ndenga BA, Mutuku FM, Ngugi HN, Mbakaya JO, Aswani P, Musunzaji PS, et al. Characteristics of Aedes aegypti adult mosquitoes in rural and urban areas of western and coastal Kenya. PLoS ONE. 2017;12:e0189971.

26. Johnson PCD, Barry SJE, Ferguson HM, Müller P. Power analysis for generalized linear mixed models in ecology and evolution. Methods Ecol Evol. 2015;6:133-42.

27. Menger DJ, Omusula P, Wouters K, Oketch C, Carreira AS, Durka M, et al. Eave screening and push-pull tactics to reduce house entry by vectors of Malaria. Am J Trop Med Hyg. 2016;94:868-78.

28. Mmbando AS, Ngowo HS, Kilalangongono M, Abbas S, Matowo NS, Moore SJ, et al. Small-scale field evaluation of push-pull system against early- and outdoor-biting malaria mosquitoes in an area of high pyrethroid resistance in Tanzania. Wellcome Open Res. 2017;2:112.

29. Wagman JM, Grieco JP, Bautista K, Polanco J, Briceno I, King R, et al. The field evaluation of a push-pull system to control malaria vectors in northern Belize. Central America. Malar J. 2015;14:184.

30. Phanitchat T, Zhao B, Haque U, Pientong C, Ekalaksananan T, et al. Spatial and temporal patterns of dengue incidence in northeastern Thailand 2006-2016. BMC Infect Dis. 2019;19:743.

31. Andrés M, Lorenz LM, Mbeleya E, Moore SJ. Modified mosquito landing boxes dispensing transfluthrin provide effective protection against Anopheles arabiensis mosquitoes under simulated outdoor conditions in a semi-field system. Malar J. 2015;14:255.

32. Amelia-Yap ZH, Chen CD, Sofian-Azirun M, Lau KW, Suana IW, Syahputra E, et al. Efficacy of mosquito coils: cross-resistance to pyrethroids in Aedes aegypti (Diptera: Culicidae) from Indonesia. J Econ Entomol. 2018;111:2854-60.

33. McPhatter LP, Mischler PD, Webb MZ, Chauhan K, Lindroth EJ, Richardson $A G$, et al. Laboratory and semi-field evaluations of two (transfluthrin) spatial repellent devices against Aedes aegypti (L.) (Diptera: Culicidae). US Army Med Dep J. 2017;1-17:13-22.

34. Wagman JM, Achee NL, Grieco JP. Insensitivity to the spatial repellent action of transfluthrin in Aedes aegypti: a heritable trait associated with decreased insecticide susceptibility. PLoS Negl Trop Dis. 2015;9:e0003726.

35. Ferreira de Ázara TM, Degener CM, Roque RA, Ohly JJ, Geier M, Eiras AE, et al. The impact of $\mathrm{CO}_{2}$ on collection of Aedes aegypti (Linnaeus) and Culex quinquefasciatus Say by BG-Sentinel ${ }^{\circledR}$ traps in Manaus, Brazil. Mem Inst Oswaldo Cruz. 2013;108:229-32.

36. Hawkes FM, Dabiré RK, Sawadogo SP, Torr SJ, Gibson G. Exploiting Anopheles responses to thermal, odour and visual stimuli to improve surveillance and control of malaria. Sci Rep. 2017;7:1728.

37. Barrera R, Amador M, Acevedo V, Caban B, Felix G, Mackay AJ. Use of the CDC autocidal gravid ovitrap to control and prevent outbreaks of Aedes aegypti (Diptera: Culicidae). J Med Entomol. 2014;51:145-54.

38. Salazar FV, Achee NL, Grieco JP, Prabaripai A, Ojo TA, Eisen L, et al. Effect of Aedes aegypti exposure to spatial repellent chemicals on BG-Sentinel trap catches. Parasit Vectors. 2013;6:145.
39. Maia MF, Kreppel K, Mbeyela E, Roman D, Mayagaya V, Lobo NF, et al. A crossover study to evaluate the diversion of malaria vectors in a community with incomplete coverage of spatial repellents in the Kilombero Valley, Tanzania. Parasit Vectors. 2016;9:451.

40. Curtis CFLJ, ljumba J, Callaghan A, Hill N, Karimza MA. The relative efficacy of repellents against mosquito vectors of disease. Med Vet Entomol. 1987;1:109-19.

41. WHO. WHO specifications for pesticides used in public health - transfluthrin. Geneva: World Health Organization; 2006. https://www.who.int/ neglected_diseases/vector_ecology/pesticide-specifications/newspecif/ en/. Accessed 13 Sep 2016.

42. Maia MF, Abonuusum A, Lorenz LM, Clausen PH, Bauer B, Garms R, et al. The effect of deltamethrin-treated net fencing around cattle enclosures on outdoor-biting mosquitoes in Kumasi, Ghana. PLoS ONE. 2012;7:e45794.

43. Lalani T, Yun H, Tribble D, Ganesan A, Kunz A, Fairchok M, et al. A comparison of compliance rates with anti-vectorial protective measures during travel to regions with dengue or chikungunya activity, and regions endemic for Plasmodium falciparum malaria. J Travel Med. 2016;23:taw043.

44. Heng S, Durnez L, Gryseels C, Van Roey K, Mean V, Sambunny UK, et al. Assuring access to topical mosquito repellents within an intensive distribution scheme: a case study in a remote province of Cambodia. Malar J. 2015;14:468.

45. Maia MF, Kliner M, Richardson M, Lengeler C, Moore SJ. Mosquito repellents for malaria prevention. Cochrane Database Syst Rev. 2018;2:CD011595.

46. Sangoro O, Kelly AH, Mtali S, Moore SJ. Feasibility of repellent use in a context of increasing outdoor transmission: a qualitative study in rural Tanzania. Malar J. 2014;13:347.

47. Mohammad A, Michael E, Robert FB. Use of a geographic information system for defining spatial risk for dengue transmission in Bangladesh: role for Aedes albopictus in an urban outbreak. Am J Trop Med Hyg. 2003;69:634-40.

48. Vavassori L, Saddler A, Muller P. Active dispersal of Aedes albopictus: a mark-release-recapture study using self-marking units. Parasit Vectors. 2019;12:583.

49. Stoddard ST, Forshey BM, Morrison AC, Paz-Soldan VA, Vazquez-Prokopec GM, Astete H, et al. House-to-house human movement drives dengue virus transmission. Proc Natl Acad Sci USA. 2013;110:994-9.

\section{Publisher's Note}

Springer Nature remains neutral with regard to jurisdictional claims in published maps and institutional affiliations.

Ready to submit your research? Choose BMC and benefit from:

- fast, convenient online submission

- thorough peer review by experienced researchers in your field

- rapid publication on acceptance

- support for research data, including large and complex data types

- gold Open Access which fosters wider collaboration and increased citations

- maximum visibility for your research: over $100 \mathrm{M}$ website views per year

At BMC, research is always in progress.

Learn more biomedcentral.com/submissions 